\title{
A Note on Geometric Degree of Finite Extensions of Mappings from a Smooth Variety by
}

\author{
Marek KARAŚ
}

Presented by Andrzej BIAEYNICKI-BIRULA

Summary. Let $f: V \rightarrow W$ be a finite polynomial mapping of algebraic subsets $V, W$ of $\mathbf{k}^{n}$ and $\mathbf{k}^{m}$, respectively, with $n \leq m$. Kwieciński [J. Pure Appl. Algebra 76 (1991)] proved that there exists a finite polynomial mapping $F: \mathbf{k}^{n} \rightarrow \mathbf{k}^{m}$ such that $\left.F\right|_{V}=f$. In this note we prove that, if $V, W \subset \mathbf{k}^{n}$ are smooth of dimension $k$ with $3 k+2 \leq n$, and $f: V \rightarrow W$ is finite, dominated and dominated on every component, then there exists a finite polynomial mapping $F: \mathbf{k}^{n} \rightarrow \mathbf{k}^{n}$ such that $\left.F\right|_{V}=f$ and $\operatorname{gdeg} F \leq(\operatorname{gdeg} f)^{k+1}$. This improves earlier results of the author.

1. Introduction. Let $\mathbf{k}$ be an algebraically closed field of characteristic zero, $V, W$ be algebraic subsets of $\mathbf{k}^{n}, \mathbf{k}^{m}$, respectively, with $n \leq m$, and let $f: V \rightarrow W$ be a finite mapping. Then there exists a finite mapping $F: \mathbf{k}^{n} \rightarrow \mathbf{k}^{m}$ such that $\left.F\right|_{V}=f[10]$.

For any finite mapping $h$ the number of points in the "generic fiber" is finite. This number is called the geometric degree of $h$ and denoted gdeg $h$. It is natural to ask about relations between $\operatorname{gdeg} F$ and $\operatorname{gdeg} f$, more precisely, between $\operatorname{gdeg} f$ and $\min \{\operatorname{gdeg} F \mid F$ is a finite extension of $f\}$.

Some examples of such relations are established in [4]-[9]. In particular, in [7] it was proved that, if $V, W$ are smooth algebraic subsets of $\mathbf{k}^{n}$ with $4 k+2 \leq n$, where $k=\operatorname{dim} V=\operatorname{dim} W$ and $f: V \rightarrow W$ is a finite dominating mapping that is dominating on every irreducible component, then there exists a finite mapping $F: \mathbf{k}^{n} \rightarrow \mathbf{k}^{n}$ such that $\left.F\right|_{V}=f$ and $\operatorname{gdeg} F \leq(\operatorname{gdeg} f)^{2 k+1}$. Later in [9] it was proved that in that situation, there exists a finite mapping $F: \mathbf{k}^{n} \rightarrow \mathbf{k}^{n}$ such that $\left.F\right|_{V}=f$ and $\operatorname{gdeg} F \leq(\operatorname{gdeg} f)^{k+1}$.

2000 Mathematics Subject Classification: 14Rxx, 14R10.

Key words and phrases: finite mapping, geometric degree, extension of mappings. 
In this short note we give a further improvement of the above two results. Namely we show the following theorem.

TheOREM 1. Let $V, W \subset \mathbb{C}^{n}$ be smooth algebraic sets of the same dimension $k$, and let $f: V \rightarrow W$ be a finite dominating mapping that is dominating on every irreducible component. If $\operatorname{dim} V=\operatorname{dim} W=k$ and $3 k+2 \leq n$, then there exists a finite mapping $F: \mathbb{C}^{n} \rightarrow \mathbb{C}^{n}$ such that $\left.F\right|_{V}=f$ and

$$
\operatorname{gdeg} F \leq(\operatorname{gdeg} f)^{k+1} .
$$

The proof is a modification of the proof given in [9] for the case $4 k+2 \leq n$.

In what follows we will work with the complex number field $\mathbb{C}$, but the results are also valid over an arbitrary algebraically closed field of characteristic zero.

2. Some useful facts. For the convenience of the reader we collect in this section all facts that we will need in the proof of Theorem 1.

The first result that we need is the following one.

Theorem 2 ([7, Thm. 3.6]). Let $V \subset \mathbb{C}^{k} \times \mathbb{C}^{n}$ be an algebraic set, and let $\pi: V \rightarrow 0 \times \mathbb{C}^{n}$ be the natural projection. If $\pi: V \rightarrow \pi(V)$ is finite and dominating on every component, and $\pi(V)$ is normal, then there exists a finite mapping $\Pi: \mathbb{C}^{k} \times \mathbb{C}^{n} \rightarrow \mathbb{C}^{k} \times \mathbb{C}^{n}$ such that $\left.\Pi\right|_{V}=\pi$ and

$$
\operatorname{gdeg} \Pi \leq(\operatorname{gdeg} \pi)^{k} .
$$

Recall that a polynomial mapping $f: V \rightarrow W$ is called dominating on an irreducible component $V^{\prime} \subset V$ if $\overline{f\left(V^{\prime}\right)}$ is an irreducible component of the set $W$ (see [7]).

The next result used in the proof is the following.

Proposition 3 ([9, Corollary 3.4]). Let $V \subset \mathbb{C}^{n} \times \mathbb{C}^{m}$ be a smooth, closed algebraic subset of dimension $k$. Moreover, assume that the projection $\pi: V \ni(x, y) \mapsto(0, y) \in 0 \times \mathbb{C}^{m}$ is finite. If $n>k+1$, then there is a linear projection $\varphi: \mathbb{C}^{n} \rightarrow \mathbb{C}^{k+1}$ such that $\varphi \times\left.\operatorname{id}_{\mathbb{C}^{m}}\right|_{V}: V \rightarrow \mathbb{C}^{k+1} \times \mathbb{C}^{m}$ is an embedding.

We will also use some results concerning embeddings. Recall that a polynomial mapping $f: V \rightarrow \mathbb{C}^{n}$ is called an embedding if $f: V \rightarrow \overline{f(V)}$ is an isomorphism.

Lemma 4 (e.g. [1]). If $X \subset \mathbb{C}^{n}$ is a closed algebraic smooth set, $\operatorname{dim} X=k$ and $n>2 k+1$, then we can change coordinates so that the projection

$$
\phi: X \ni(x, y) \mapsto(0, y) \in 0 \times \mathbb{C}^{2 k+1}
$$

is an embedding. 
Theorem 5 (e.g. [2], [3] or [11]). Let $X \subset \mathbb{C}^{n}$ be a closed algebraic set which is smooth (not necessarily irreducible) of dimension (not necessarily pure) $k$. Let $\phi: X \rightarrow \mathbb{C}^{n}$ be an embedding. If $n>2 k+1$ then there exists an isomorphism $\Phi: \mathbb{C}^{n} \rightarrow \mathbb{C}^{n}$ such that

$$
\left.\Phi\right|_{X}=\phi .
$$

3. Proof of the theorem. Since $3 k+2 \leq n$, we have $n-(k+1) \geq 2 k+1$. Thus, by Lemma 4 we can assume that the projections $\varphi_{1}: V \rightarrow \mathbb{C}^{2 k+1} \times 0$ and $\varphi_{2}: W \rightarrow 0 \times \mathbb{C}^{n-k-1}$ are embeddings. For $\widetilde{V}=\varphi_{1}(V), \widetilde{W}=\varphi_{2}(W)$ the mapping $\widetilde{f}=\varphi_{2} \circ f \circ \varphi_{1}^{-1}: \widetilde{V} \rightarrow \widetilde{W}$ is finite with gdeg $\widetilde{f}=\operatorname{gdeg} f$.

Consider the sets $\widetilde{V}$ and $\widetilde{W}$ as subsets of $\mathbb{C}^{2 k+1}$ and $\mathbb{C}^{n-k-1}$, respectively. The mapping $\psi: \widetilde{V} \ni x \mapsto(x, \widetilde{f}(x)) \in \mathbb{C}^{2 k+1} \times \mathbb{C}^{n-k-1}=\mathbb{C}^{n+k}$ gives the isomorphism between $\widetilde{V}$ and $\widehat{V}=\psi(\widetilde{V})$. Since $\psi: \widetilde{V} \rightarrow \widehat{V}$ is an isomorphism, for the projection $\pi: \widehat{V} \ni(x, y) \mapsto(0, y) \in 0 \times \mathbb{C}^{n-k-1}$ we have $\widetilde{f}=\pi \circ \psi$ and $\operatorname{gdeg} \pi=\operatorname{gdeg} \tilde{f}=\operatorname{gdeg} f$.

The set $\widehat{V}=\psi\left(\varphi_{1}(V)\right)$ is smooth. Thus, by Proposition 3 applied to the mapping $\pi: \widehat{V} \rightarrow 0 \times \mathbb{C}^{n-k-1}$, there is a projection $\varphi: \mathbb{C}^{2 k+1} \rightarrow$ $0 \times \mathbb{C}^{k+1}$ such that $\varphi \times \mathrm{id}_{\mathbb{C}^{n-k-1}}: \mathbb{C}^{2 k+1} \times \mathbb{C}^{n-k-1} \rightarrow\left(0 \times \mathbb{C}^{k+1}\right) \times \mathbb{C}^{n-k-1}$ restricted to the set $\widehat{V}$ is an embedding. Let us write $\widetilde{\varphi}$ for the mapping $\left.\left(\varphi \times \operatorname{id}_{\mathbb{C}^{n-2 k-1}}\right)\right|_{\widehat{V}}$. Because $V_{2}=\widetilde{\varphi}(\widehat{V}) \subset\left(0 \times \mathbb{C}^{k+1}\right) \times \mathbb{C}^{n-k-1}$ we can consider this set as a subset of $\mathbb{C}^{k+1} \times \mathbb{C}^{n-k-1}=\mathbb{C}^{n}$. Then for the mapping $\pi_{2}$ : $V_{2} \ni(x, y) \mapsto(0, y) \in 0 \times \mathbb{C}^{n-k-1}$ we have $\pi=\pi_{2} \circ \widetilde{\varphi}, \pi_{2}$ is finite and $\operatorname{gdeg} \pi_{2}=\operatorname{gdeg} \pi$. Since $\pi_{2}\left(V_{2}\right)=\widetilde{W}$ is smooth, by Theorem 2 there is a finite mapping $\Pi_{2}: \mathbb{C}^{k+1} \times \mathbb{C}^{n-k-1} \rightarrow \mathbb{C}^{k+1} \times \mathbb{C}^{n-k-1}$ such that $\left.\Pi_{2}\right|_{V_{2}}=\pi_{2}$ and $\operatorname{gdeg} \Pi_{2} \leq\left(\operatorname{gdeg} \pi_{2}\right)^{k+1}=(\operatorname{gdeg} f)^{k+1}$.

Now by Theorem 5 applied to the mappings $\varphi_{1}: V \rightarrow \widetilde{V}, \widetilde{\varphi} \circ \psi: \widetilde{V} \rightarrow V_{2}$ and $\varphi_{2}: W \rightarrow \widetilde{W}$ there are isomorphisms $\Phi_{1}, \Psi, \Phi_{2}: \mathbb{C}^{n} \rightarrow \mathbb{C}^{n}$ such that $\left.\Phi_{1}\right|_{V}=\varphi_{1},\left.\Psi\right|_{\widetilde{V}}=\widetilde{\varphi} \circ \psi$ and $\left.\Phi_{2}\right|_{W}=\varphi_{2}$

Putting $F=\Phi_{2}^{-1} \circ \Pi_{2} \circ \Psi \circ \Phi_{1}$ we have a finite extension of $f$ such that $\operatorname{gdeg} F \leq(\operatorname{gdeg} f)^{k+1}$.

\section{References}

[1] Z. Jelonek, The extension of regular and rational embeddings, Math. Ann. 277 (1987), 113-120.

[2] - A note about the extension of polynomial embeddings, Bull. Polish Acad. Sci. Math. 43 (1995), 239-244.

[3] S. Kaliman, Extension of isomorphisms between affine algebraic subvarieties of $k^{n}$ to automorphisms of $k^{n}$, Proc. Amer. Math. Soc. 113 (1991), 325-334.

[4] M. Karaś, An estimation of the geometric degree of an extension of some polynomial proper mappings, Univ. Iagell. Acta Math. 35 (1997), 131-135. 
[5] M. Karaś, Geometric degree of finite extension of projections, ibid. 37 (1999), 109119 .

[6] —, Birational finite extensions, J. Pure Appl. Algebra 148 (2000), 251-253.

[7] -, Finite extensions of mappings from a smooth variety, Ann. Polon. Math. 75 (2000), 79-86.

[8] - Finite extensions of mappings of finite sets, Bull. Polish Acad. Sci. Math. 50 (2002), 239-241.

[9] - Geometric degree of finite extension of mappings from a smooth variety, J. Pure Appl. Algebra 212 (2008), 1145-1148.

[10] M. Kwieciński, Extending finite mappings to affine space, ibid. 76 (1991), 151-153.

[11] V. Srinivas, On the embedding dimension of the affine variety, Math. Ann. 289 (1991), 125-132.

Marek Karaś

Instytut Matematyki

Uniwersytet Jagielloński

Reymonta 4

30-059 Kraków, Poland

E-mail: Marek.Karas@im.uj.edu.pl 